\title{
La coopération suisse au développement dans le contexte international
}

L'aide publique suisse au développement vue par l'OCDE et l'évolution de la notion d'« aide publique au développement » au sein de l'OCDE

\section{Thomas Greminger}

\section{CpenEdition}

\section{Journals}

Édition électronique

URL : http://journals.openedition.org/aspd/1476

DOI : $10.4000 /$ aspd. 1476

ISSN : 1663-9669

Éditeur

Institut de hautes études internationales et du développement

Édition imprimée

Date de publication : 1 février 1995

Pagination : 233-242

ISSN : 1660-5934

Référence électronique

Thomas Greminger, «La coopération suisse au développement dans le contexte international », Annuaire suisse de politique de développement [En ligne], 14 | 1995, mis en ligne le 04 mai 2013, consulté le 08 septembre 2020. URL : http://journals.openedition.org/aspd/1476 ; DOI : https:// doi.org/10.4000/aspd. 1476 


\title{
La coopération suisse au développement dans le contexte international
}

\section{L'aide publique suisse au développement vue par l'OCDE et l'évolution de la notion d'"aide publique au développement" au sein de l'OCDE}

\author{
Thomas Greminger, DDA
}

\section{Introduction}

Où se situe la coopération suisse au développement dans le contexte international? Nous voulons aborder cette question du point de vue du Comité d'aide au développement (CAD) de l'OCDE qui examine régulièrement les prestations fournies dans ce domaine par ses membres. Pour cela nous avons analysé les principaux éléments quantitatifs et qualitatifs des critiques émises lors des trois derniers examens de l'aide suisse (1988, 1990 et 1993). Les considérations quantitatives et la notion qui les accompagne d'une répartition internationale équitable du poids de l'aide fournie (“burden sharing") sont bien entendu au centre de l'évaluation de l'OCDE. Nous devons donc aussi accorder une place prépondérante à l'aspect quantitatif.

Dans ce contexte, la question relative à la comparabilité des statistiques sur les prestations fournies est pour ainsi dire incontournable. II n'est pas nécessaire d'être spécialiste pour savoir que certains pays interprètent de façon plus large que d'autres les directives statistiques à appliquer dans plusieurs secteurs, comme l'aide aux réfugiés ou les actions de désendettement. Cette inégalité dans l'application des directives, mais aussi l'apparition de nouvelles formes de coopération au développement telles que le soutien des processus de démocratisation ou de démobilisation, les fonds pour l'environnement qui visent aussi la promotion du Sud (le Fonds mondial pour l'environnement par 
exemple), de même que l'existence de nouvelles catégories de pays bénéficiaires en Europe centrale et de l'Est et dans l'ex-Union soviétique, ont incité I'OCDE en 1991 à revoir sa notion de l'aide publique au développement (APD ou Official Development Aid = ODA) tant du point de vue géographique que sectoriel. Trois ans plus tard, on constate que des solutions se dessinent dans quelques domaines, tandis que les travaux se poursuivent encore dans d'autres. La deuxième partie du présent article tire donc un premier bilan de cette évolution et examine les conséquences de ces mesures sur l'évaluation des prestations suisses en matière d'APD.

\section{L'aide publique suisse au développement vue par l'OCDE}

\section{Remarques récurrentes}

Une analyse des examens par pays de 1988, 1990 et 1993 - le nombre de ses membres ayant augmenté, le Comité d'aide au développement de l'OCDE a étendu de deux à trois ans la durée séparant deux examens - montre d'emblée qu'une série de thèmes reviennent sans cesse, à des degrés divers selon les rapports, dans la critique adressée à la Suisse. Le principal reproche, plus ou moins véhément, concerne en général le volume de l'aide publique suisse au développement. Le parallèle tiré entre la modestie des prestations quantitatives et la qualité élevée de l'aide, universellement reconnue, est une autre constante de la critique. Pour ce qui est de la qualité, les examens louent régulièrement l'importance de l'aide non liée, le fait que l'aide suisse est accordée à titre de don, le désintérêt politique et la volonté de faire participer la population du pays bénéficiaire, tandis qu'ils reprochent à la Suisse une concentration insuffisante de l'aide aussi bien en 1990 qu'en 1993. Nous commençons par nous pencher sur ces aspects récurrents des examens du CAD pour aborder ensuite quelques remarques plus spécifiques qui n'apparaissent pas dans tous les rapports d'examen.

\section{Volume insatisfaisant}

En 1987 et en 1989, le volume de l'aide publique suisse au développement a atteint respectivement 0,31 et $0,30 \%$ du PNB. La Suisse se situait ainsi endessous de la moyenne pondérée des pays du CAD de 0,33\% et même nettement en-dessous de la moyenne non pondérée de 0,51\% (1989), car le niveau relativement bas des dépenses des deux principaux donneurs du point de vue nominal, les Etats-Unis $(0,15 \%)$ et le Japon $(0,31 \%)$, abaisse sensiblement la moyenne pondérée. Le CAD a donc demandé à la Suisse de se fixer un objectif plus ambitieux qui corresponde davantage à sa capacité économique, à ses relations commerciales extérieures (le CAD relève que la balance commerciale de la Suisse avec les pays en développement est largement excédentaire) et à sa tradition humanitaire. Le dernier examen, qui date de 1993, concerne les chiffres exceptionnels de 1992. Les coûts liés à l'adhésion aux institutions de Bretton Woods, qui comprennent des versements uniques aux filiales de la Banque mondiale que sont l'AID et la SFI et une participation au capital de la 
Banque mondiale (190 millions de francs au total), ont accru le volume de l'aide qui a ainsi atteint $0,39 \%$ du PNB selon le mode de calcul suisse. Selon les directives statistiques de l'OCDE, I'APD suisse a même atteint $0,46 \%$ du PNB en 1992. La différence s'explique par le fait que l'OCDE comptabilise les contributions aux banques de développement au moment de la fixation du change, tandis que la Suisse ne les consigne que lorsqu'elles sont effectivement versées. La pratique discutable de l'OCDE comptabilise ainsi en 1992 toute la participation au capital de la Banque mondiale (somme totale à verser: 295,8 millions de francs) à titre d'aide publique au développement, tandis que la statistique suisse n'a en fait comptabilisé en 1992 que le cinquième de cette somme, soit le montant effectivement versé. Quoi qu'il en soit, la Suisse a ainsi pour la première fois dépassé la moyenne pondérée du CAD et s'est placée dans le peloton de tête des pays donneurs. Le CAD a enregistré cette progression avec beaucoup de satisfaction. Le Comité a toutefois reconnu que les planifications budgétaire et financière à court et à moyen terme laissaient présager un avenir moins réjouissant. C'est ce qui l'a incité à “inviter les autorités suisses à entreprendre tous les efforts nécessaires pour respecter l'engagement pris à la CNUED, à Rio de Janeiro, d'atteindre l'objectif de $0,40 \%$ du PNB avant l'an 2000" et à leur rappeler le principe d'une "meilleure répartition internationale du poids de l'aide fournie".' Comme d'autres rapports d'examen l'avaient fait précédemment, le dernier indique comme référence réaliste la moyenne des pays de l'Union européenne $(0,44 \%$ du PNB en 1992).

Dans la pratique, les dépenses d'APD de la Suisse ont enregistré en 1993 le recul attendu pour se situer à $0,34 \%$ du PNB selon les calculs suisses et à $0,32 \%$ selon l'OCDE (en raison de la différence du mode de comptabilisation évoquée ci-dessus). Comme le souligne le communiqué de presse de l'OCDE', la Suisse affiche pour 1993 la plus forte baisse en volume des membres du $\mathrm{CAD}$ après la Finlande. Alors que le plan financier en vigueur au moment de l'examen (24.9.1993) donnait à penser que le volume de l'aide suisse allait s'accroître progressivement pour atteindre $0,37 \%$ du PNB en 1997, de nouvelles restrictions dues aux problèmes budgétaires de la Confédération ont provoqué une nette correction à la baisse de ces prévisions. Peu avant les vacances d'été 1994, le Conseil fédéral a décidé de "plafonner" jusqu'à nouvel avis (budget 1995, plan financier 1996-1998) l'aide au développement de la Confédération à $0,34 \%$ du PNB. Le besoin croissant de réduire les dépenses vient même de l'obliger à abandonner ce "plafond" dans le plan financier. A l'avenir, l'aide devrait certes continuer d'enregistrer un accroissement réel modeste, mais son volume en pourcentage du PNB ne pourra guère se maintenir à $0,34 \%$ et encore moins s'approcher du $0,40 \%$ du PNB préconisé par l'OCDE.

II n'en reste pas moins que le $0,40 \%$ du PNB demeure l'objectif déclaré du Conseil fédéral, comme il l'indique dans son récent "Rapport sur les relations Nord-Sud de la Suisse dans les années 90 (Lignes directrices Nord-Sud)". Les prévisions relatives au volume de l'aide pour l'ensemble des pays membres du CAD ne sont par ailleurs pas plus encourageantes: il ne sera pas possible de compenser rapidement la forte baisse qu'a subie la moyenne pondérée du CAD 
qui est tombée à $0,29 \%$ du PNB en 1993. Au contraire, on pourrait s'attendre à un nouveau recul, car la tendance semble indiquer une stabilisation réelle des budgets de l'aide. Ces perspectives laissent présager que la Suisse conservera à l'avenir sa place dans la moyenne des donneurs internationaux, mais ne pourra sans doute pas l'améliorer sensiblement. L'écart entre l'APD suisse et la moyenne de l'Union européenne devrait également demeurer constant, tout comme la différence entre l'aide et la capacité économique de notre pays (revenu par habitant le plus élevé de tous les pays de l'OCDE).

\section{Qualité et contribution des oeuvres d'entraide}

A ces critiques, on répond volontiers du côté suisse en soulignant la qualité remarquable de la coopération suisse au développement et l'importance de la contribution des oeuvres d'entraide suisses qui complètent efficacement l'aide publique au développement. II est vrai que ces deux arguments sont invoqués à juste titre et le CAD le reconnaît. Pour ce qui esi du volume de l'aide fournie par les oeuvres d'entraide privées, la Suisse se situe en effet au deuxième rang ${ }^{3}$, bien que ce fait ne saurait corriger les données quantitatives de l'aide publique.

\section{La part de l'aide liée est minime}

La part de l'aide liée constitue certainement un indicateur important de la qualité de l'aide. Les membres du CAD sont largement d'accord sur le fait que l'aide liée débouche en général sur de moins bons résultats que l'aide non liée, car le donneur accorde plus d'importance, dans le cadre de la première, à la promotion de ses propres exportations qu'aux besoins de développement du bénéficiaire. Dans l'aide bilatérale suisse, la part de l'aide liée est minime, puisqu'en principe seuls les crédits mixtes (part fédérale en 1992: 83,2 millions de francs) et une partie de l'aide alimentaire (29,5 millions de francs en 1992) sont liés à des livraisons suisses. Dans l'aide de toute une série de pays donneurs (Australie, Belgique, Italie, Espagne), une part de plus de 50\% est liée ${ }^{4}$. Mais cela ne signifie nullement que la coopération au développement n'ait que peu d'effets sur l'économie suisse: en 1992, environ 1,5 milliards de francs de la coopération internationale au développement auraient été versés à des Suisses et à des entreprises suisses ${ }^{5}$. Une bonne partie de ces versements sont à mettre sur le compte de la capacité concurrentielle internationale des entreprises suisses de l'industrie et des services.

\section{Part des dons, désintérêt politique et participation des bénéficiaires}

Le Comité d'aide au développement relève avec satisfaction que l'aide suisse est exclusivement accordée à titre de don depuis 1988. La Suisse évite ainsi que l'aide au développement ne contribue à aggraver l'endettement des pays bénéficiaires. La moitié seulement des membres du CAD accordent l'ensemble de leur aide sous forme de don, tandis que nombre de pays (Autriche, Italie, Japon, Espagne) allouent plus des deux tiers de leur aide sous forme de crédits. Les pays bénéficiaires apprécient et le CAD reconnaît comme preuve de qualité le fait que la Suisse, en tant que petit pays sans prétentions colonia- 
les, fournit son aide en l'absence de toute visée politique nationale ou régionale. La part relativement importante de l'aide que la Suisse accorde aux pays classés parmi les plus pauvres (pays les moins développés, pays à faible revenu) constitue une preuve de son désintérêt politique. En 1993 par exemple, seuls $19 \%$ de l'aide ont été accordés à des pays dont le revenu par habitant est supérieur à 675 dollars. Lorsque l'on mène une politique de développement désintéressée, il est plus facile de s'engager de façon cohérente et crédible en faveur d'une meilleure "gestion des affaires publiques" dans le pays partenaire. C'est également pour cette raison que la Suisse est parvenue, avant d'autres pays et avec plus d'efficacité, à orienter sa coopération au développement vers une forme plus participative. Cette orientation vise à faire participer les groupes de population concernés à la planification et à la réalisation de projets et de programmes. Le CAD a relevé cette qualité dans ses examens de 1990 et de 1993. L'utilisation conforme aux lignes directrices des crédits mixtes ainsi que le rôle novateur de la Suisse dans la création d'un instrument de désendettement comptent parmi les autres éléments que le Comité d'aide au développement a loués à l'occasion de plus d'un examen.

\section{Manque de concentration de l'aide bilatérale}

Dans les deux derniers examens, c'est le manque de concentration de la coopération au développement bilatérale qui soulève les plus vives critiques. Selon le Secrétariat du CAD, 104 pays en développement auraient reçu un soutien de la Suisse en $1991 / 1992^{6}$. Tandis que ce nombre, par trop élevé, n'a guère évolué depuis le début des années 80 (99 en 1981/1982), une comparaison annuelle des vingt principaux bénéficiaires démontre un net accroissement du manque de concentration de l'aide bilatérale (aide humanitaire comprise). En $1981 / 1982,74 \%$ de l'aide bilatérale ont été adressés aux vingt premiers pays, qui ne correspondent par ailleurs pas tout à fait aux pays dits "de concentration". Cinq ans plus tard, cette part se montait à $70 \%$ et n'atteignait plus que $62 \%$ en $1991 / 1992$. Selon la recommandation du CAD, la Suisse, en tant que donneur modeste, aurait tout intérêt à réduire le nombre de bénéficiaires de son aide et à rationaliser la composition du groupe des principaux bénéficiaires. La volonté, exprimée dans le récent Message concernant la continuation de la coopération technique et de l'aide financière en faveur des pays en développement, de réduire à moyen terme le nombre des pays de concentration et les efforts fournis par la DDA et l'Office fédéral des affaires économiques extérieures (OFAEE) pour mieux coordonner la programmation par pays, indique que ces critiques sont prises au sérieux par les responsables concernés. Mais ceuxci relèvent également, par exemple dans le "Mémorandum" à l'OCDE concernant la coopération suisse au développement ${ }^{7}$, que les efforts de concentration ont des limites. Celles-ci apparaissent clairement dans le cadre des contributions aux programmes des oeuvres d'entraide, où l'administration centrale n'intervient pas dans le choix des pays bénéficiaires. Mais elles existent aussi dans le cas d'instruments qui ne peuvent, de par leur nature, s'intégrer dans la politique de concentration. II s'agit par exemple des mesures de désendette- 
ment, puisque l'existence de titres de créance pouvant être rachetés à des créanciers suisses joue un rôle clé dans le choix des pays.

\section{De la politique démographique à la cohérence}

D'autres aspects ayant suscité des critiques de la part du Comité d'aide au développement méritent d'être mentionnés. En 1990, c'est par exemple l'engagement de la Suisse au niveau de la politique démographique qui était jugé trop modeste. En effet, la politique démographique dans son sens restreint, c'est-àdire lorsqu'elle se limite aux programmes de planification familiale, n'est pas et n'a jamais été un secteur important de la coopération bilatérale suisse au développement. Si l'on considère la politique démographique dans un sens plus large, c'est-à-dire l'engagement en faveur d'une amélioration de l'approvisionnement destinée aux soins de santé primaires, l'encouragement de l'éducation de base et de la formation des femmes, le bilan de la Suisse apparaît nettement moins négatif. De plus, la Suisse alloue des contributions croissantes à des institutions internationales oeuvrant dans ce domaine. C'est le cas par exemple pour le Fonds des Nations unies pour les activités en matière de population (FNUAP) et pour la Fédération internationale pour la planification familiale (IPPF).

En 1993, ce sont l'élaboration interne de thèmes horizontaux et politiques, le travail d'information et les problèmes de cohérence politique qui ont été abordés. Le Comité d'aide au développement a ainsi cru déceler des faiblesses au niveau des thèmes dits "horizontaux" qui se retrouvent dans tous les domaines sectoriels et géographiques, par exemple le rôle des femmes dans le développement. Pour ce qui est du thème "Femmes et développement", le $\mathrm{CAD}$ a relevé à juste titre que les effectifs consacrés à ce domaine sont nettement moins nombreux en Suisse que dans d'autres pays donneurs. Depuis lors, cette situation a été corrigée. De manière générale, le CAD a recommandé à l'aide publique au développement (APD) suisse d'accroître ses moyens et son personnel pour traiter des questions politiques et pour mieux appliquer les décisions y relatives à la centrale et dans les bureaux de coordination.

Le budget du Service d'information de la DDA représente $0,21 \%$ de l'APD suisse. C'est ce qui est investi pour l'information et la formation au niveau du public et des écoles en Suisse. Malgré le rôle joué par les oeuvres d'entraide suisses dans ce domaine et malgré la méfiance des Suisses face à l'information fournie par l'Etat, les membres du CAD sont persuadés que la Suisse devrait fournir un travail plus important dans ce secteur: il est indispensable, et pas seulement en Suisse, d'accroître les activités d'information et de formation sur les nouveaux aspects, plus globaux, des relations Nord-Sud. Ces activités ne doivent pas avoir pour seul objectif de libérer plus de fonds pour le développement, mais surtout de faire comprendre qu'il importe d'appliquer une politique plus cohérente face aux pays en développement.

En considérant l'ensemble des relations de notre pays avec les pays en développement, le Comité pense également qu'une meilleure cohérence est nécessaire entre l'APD et les autres secteurs de la politique. Dans son commu- 
niqué de presse, il cite explicitement le commerce et l'agriculture, mais le rapport d'examen évoque également la fuite de capitaux et le blanchiment d'argent sale. Naturellement, les problèmes de cohérence constituent un défi de taille pour pratiquement tous les pays de l'OCDE. II convient donc désormais de passer d'une politique de la coopération au développement à une politique de développement conséquente et intersectorielle. Cette question de cohérence, le Conseil fédéral l'a abordée dans son “Rapport sur les relations NordSud de la Suisse dans les années 90 (Lignes directrices Nord-Sud)" et il s'agit à présent d'appliquer ce principe dans la réalité politique.

\section{La notion "d'aide publique au développement" de l'OCDE évolue - conséquences pour la Suisse}

Bien que les critères qualitatifs aient gagné en importance, les considérations quantitatives, c'est-à-dire celles relatives aux montants de l'aide publique au développement, continueront de jouer un rôle central dans l'appréciation de l'OCDE. De même, la "répartition internationale équitable des charges" continuera d'être perçue du point de vue quantitatif. II est donc d'autant plus crucial de savoir ce qu'il convient de considérer comme "aide publique au développement". Plusieurs éléments conjoints - modes de comptabilisation différents selon les pays membres, apparition de nouveaux pays bénéficiaires après l'effondrement du bloc de l'Est et création de nouvelles formes de coopération au développement - ont provoqué une évolution de la notion de l'APD admise jusqu'alors et suscité dès 1991 un processus de réforme. Cette réforme a une dimension géographique, qui se concrétise dans la réorganisation de la liste du $\mathrm{CAD}$ des pays en développement, et une dimension sectorielle, qui concerne les formes d'APD nouvelles ou controversées. Dans ces deux domaines, la discussion paraît au premier abord surtout technique, mais la durée de la réforme témoigne à elle seule de l'importance politique de bon nombre des questions abordées. Certaines étapes de la réforme engendreront d'importants changements dans la pratique de comptabilisation de quelques Etats membres - avec tous les effets que cela implique sur le volume de l'aide - c'est pourquoi certains campent souvent sur leurs positions.

\section{Esquisse d'une nouvelle liste des pays en développement}

En ce qui concerne la redéfinition d'une liste du CAD des pays en développement, on serait tenté de citer le cas de la montagne qui a accouché d'une souris, bien que nul ne conteste la nécessité de revoir d'urgence une liste désuète et historiquement dépassée sur laquelle figurent encore des pays tels que Singapour, Israël et la Grèce. La difficulté de ce travail ne réside pas uniquement dans les relations particulières de certains pays de l'OCDE avec certains "pays en développement", mais aussi dans leur portée: il s'agit en fait rien moins que de formuler une définition des pays en développement. Un consensus a cependant pu être trouvé: on recense tous les bénéficiaires de flux d'aide concessionnelle, tous n'étant pas des pays en développement. La 
liste a donc été séparée en deux parties: la première comprend les pays en développement les moins avancés selon leur revenu par habitant et la deuxième tous les autres pays destinataires d'une aide. Ne peut être comptabilisée comme "aide publique au développement" que l'aide accordée aux pays de la première partie de cette liste ${ }^{8}$.

Reste à déterminer où se situe la limite entre les deux parties de la liste, quels critères adopter pour admettre ou exclure un pays de la liste II et si les pays d'Europe centrale et de l'Est doivent bénéficier du même traitement ou non que les pays en développement traditionnels. Pendant longtemps, un consensus concernant l'exclusion semblait se dessiner autour de la limite imposée par la Banque mondiale pour l'octroi de ses prêts (revenu par habitant de 4715 dollars américains en 1992). En raison des intérêts particuliers d'un important donneur, la rencontre ministérielle des pays du CAD, en 1993, n'a toutefois pu fixer que le montant de 8355 dollars comme limite. II n'en reste pas moins que, dès 1996, l'aide accordée aux "pays à haut revenu», tels qu'lsraël, Chypre ou Taïwan, ne pourra plus être comptabilisée dans l'APD. II y a quelque temps déjà, lors de la procếdure d'évaluation cas par cas, le CAD avait décidé d'exclure dès 1996 les cinq pays à haut revenu les plus nantis (Bahamas, Singapour, Koweït, Emirats arabes unis, Qatar) de la liste des pays en développement et d'y admettre immmédiatement les anciennes républiques soviétiques d'Asie centrale et du Cáucase ${ }^{9}$.

\section{Efforts de démocratisation et de démobilisation, lutte contre la drogue: soutien incontesté}

Au niveau sectoriel, toute une série de nouvelles formes d'aide ont pu être admises, pratiquement sans opposition, dans le règlement de comptabilisation. Cette innovation n'a posé aucune difficulté car elle équivalait à officialiser une pratique déjà courante. Selon les nouvelles directives de l'OCDE, il est désormais possible de comptabiliser à titre d'aide publique au développement le soutien accordé aux processus de démocratisation et de démobilisation. La première de ces notions recouvre par exemple l'envoi de missions d'observation lors d'élections ou l'aide technique destinée à l'enregistrement et à la formation d'électeurs, la seconde comprend, entre autres, des programmes de réinsertion pour les soldats démobilisés. En matière de lutte contre la drogue, des précisions ont été apportées au règlement existant pour que ne puissent être comptabilisés que les programmes visant à promouvoir le développement. Les programmes répressifs, tels que le financement d'interventions de la police ou de l'armée pour détruire les cultures concernées, n'entrent donc pas en ligne de compte.

Mesures de maintien de la paix, aide aux réfugiés, Fonds pour l'environnement, actions de désendettement: soutien controversé

En quelques années à peine, les dépenses consacrées aux maintien de la paix ont fortement augmenté et provoqué une démobilisation dangereuse dans le domaine de l'aide publique au développement. C'est ce qu'a écrit à juste titre 
Gus Speth, administrateur du PNUD, dans une lettre, qui a suscité quelques remous, récemment adressée aux chefs des agences de développement bilatéral. D'aucuns seraient tentés de mettre la charrue avant les boeufs, c'est-à-dire de décréter que la totalité ou une partie importante des dépenses consacrées au maintien de la paix peuvent être comptabilisées dans l'APD. Une proposition allant dans ce sens de quelques membres du CAD a toutefois été balayée par la grande majorité du Comité et n'a guère de chances de s'imposer à l'avenir. Le CAD a néanmoins été chargé d'enregistrer, à titre indicatif, les dépenses consenties par ses membres en faveur du maintien de la paix, mais en dehors de l'APD, afin de disposer d'une base de données fiable.

Dans le domaine de l'aide aux réfugiés, le règlement actuel permet dans une certaine mesure de comptabiliser dans l'APD les dépenses consenties dans le pays donneur pour la prise en charge de réfugiés. La plupart des membres du CAD ne font toutefois pas usage de cette possibilité, car de telles dépenses n'ont de toute évidence rien de commun avec l'aide au développement. Un groupe de pays, qui ne cesse de rétrécir - il ne comprend aujourd'hui plus que l'Allemagne, l'Autriche, les Pays-Bas, le Danemark et la Finlande - continue cependant de compter de tels frais dans I'APD. Sur les instances de divers pays, dont la Suisse, une proposition a été présentée pour supprimer définitivement ce principe de comptabilisation tout en prévoyant un délai transitoire de plusieurs années.

Dans le cadre de fonds globaux pour l'environnement - dont les principaux organismes sont le Fonds mondial pour l'environnement (FME) et le Protocole de Montréal - un compromis a désormais été trouvé. II pourrait mettre un terme aux tiraillements qui ont divisé pendant trois ans les membres du CAD au sujet de la possibilité de comptabiliser ces dépenses dans l'APD et qui permettrait notamment d'éviter l'application de modes de calcul différents qui rendent toujours plus difficile la comparaison entre les statistiques de l'APD. La solution proposée séduit par sa simplicité: à l'avenir, $50 \%$ des contributions à des fonds globaux pour l'environnement devraient pouvoir être comptabilisés dans l'APD.

En matière de comptabilisation des actions de désendettement, le CAD n'a pas encore trouvé un compromis. La directive actuelle permet de compter comme aide publique au développement la valeur nominale d'une dette remise, même si la valeur réelle de cette dette ne représente plus qu'une part infime de la valeur nominale et que la remise pèse d'un poids beaucoup moins grand sur le budget d'APD du pays donneur. Se fondant sur ces réflexions, les Pays-Bas et la Suisse en particulier, plaident en faveur d'une adaptation du règlement afin que seul le montant réel de la remise puisse être comptabilisé au titre d'APD.

\section{Conséquences pour le volume de l'APD suisse}

Tout au long du processus de réforme, la Suisse a préconisé qu'il convenait d'une part d'adapter la notion d'aide publique au développement aux circonstances et évolutions nouvelles, afin qu'elle puisse continuer de servir d'étalon de mesure pour la "répartition internationale des charges", mais elle a d'autre part souligné qu'il fallait éviter de diluer la notion d'APD et ne pas renoncer au 
critère qu'est le développement pour l'orientation de l'aide. L'application d'une politique de comptabilisation "favorable au budget" - euphémisme utilisé par un membre du CAD pour désigner la tendance consistant à inscrire le maximum des postes du budget dans l'APD - n'est pas un objectif de la Suisse. II n'en reste pas moins intéressant d'analyser les conséquences des réformes entreprises pour le volume d'aide que la Suisse peut comptabiliser à titre d'APD.

Les effets diffèrent selon la définition adoptée: la formule utilisée par la DDA et l'OFAEE dans leur rapport annuel, la définition de l'OCDE ou la rubrique du budget fédéral. Dans tous les cas, ces effets demeurent toutefois modestes. Dans la statistique de l'OCDE pour 1993, la Suisse aurait ainsi pu inscrire 20 millions de francs de plus à titre d'APD. Ce supplément est à mettre pour moitié sur le compte des anciennes républiques soviétiques d'Asie (10,8 millions) et sur les contributions suivantes: 8,5 millions à des fonds globaux pour l'environnement ( 7,3 millions de francs au FME; 1,2 millions au Fonds pour l'ozone du Protocole de Montréal), 1,4 millions pour les missions d'observation lors d'élections et 0,9 million destiné au Fonds des Nations Unies pour la lutte anti-drogue (FNULAD). A quelques nuances près - des nuances minimes dues à des différences techniques (date de comptabilisation) - ces dépenses correspondent également à l'image fournie dans le rapport annuel 1993 de la DDA et de I'OFAEE. Comme indiqué plus haut, la principale différence entre ces deux définitions provient du fait que les contributions aux banques de développement ne sont pas comptabilisées au même moment. Cette règle peu judicieuse comme le concède le Secrétariat du CAD lui-même - ne sera sans doute pas modifiée dans un avenir proche, car les membres du Comité semblent loin d'un compromis à ce propos.

La rubrique "coopération publique au développement" du budget fédéral n'a pas encore été adaptée aux réformes opérées par le CAD. Puisque la plupart des effets s'annulent réciproquement, les différences qui en résultent demeurent minimes. A long terme, une meilleure transparence exigera toutefois sans doute que cette rubrique soit elle aussi adaptée pour éviter que l'on confonde diverses indications du volume de l'aide.

\section{Notes}

1. DAC Aid Review of Switzerland, OCDE, Communiqué de presse, Paris, 28.9.1993.

2. La structure des apports financiers aux pays en développement et aux pays en transition subit de profondes modifications, OCDE, Communiqué de presse, Paris 20.6.1994.

3. CAD/OCDE: Coopération pour le développement - Rapport 1993, Paris 1994, p. 163.

4. The Reality of Aid '94, édité par ICVA, EUROSTEP et ACTIONAID, 1994, p. 28.

5. Greminger, Thomas: OECD examiniert schweizerische Entwicklungszusammenarbeit, dans: Volkswirtschaft 2/94, pp. 50/51/tab. 5.

6. CAD/OCDE: Examen de l'aide 1993/94; Rapport du secrétariat et questions pour l'examen de la Suisse, DCD/DAC/AR(93)2/19, 3.9.1993, p. 21 et tab. 6.

7. DDAVOFAEE: Mémorandum de la Suisse au Comité d'aide au développement de l'OCDE 1992, 16.7.1993, pp. 22/23.

8. Cf. Rapport du CAD 1993 (note 3), pp. 11-13.

9. Arménie, Géorgie, Tadjikistan, Azerbaïdjan, Kirghizistan, Ouzbékistan, Turkménistan, Kazakhstan. 